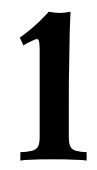

\title{
EVALUACIÓN DE COMPETENCIAS PROFESIONALES EN EDUCACIÓN SUPERIOR: RETOS E IMPLICACIONES
}

\author{
(EVALUATION OF PROFESSIONAL COMPETENCES IN HIGHER EDUCATION: \\ CHALLENGES AND IMPLICATIONS)
}

José Tejada Fernández

Carmen Ruiz Bueno

Universidad Autónoma de Barcelona

DOI: $10.5944 / e d u c X X 1.12175$

\section{Cómo referenciar este artículo/How to reference this article:}

Tejada Fernández, J. y Ruiz Bueno, C. (2016). Evaluación de competencias profesionales en Educación Superior: Retos e implicaciones. Educación XX1, 19(1), 17-38, doi:10.5944/educXX1.12175

Tejada Fernández, J. \& Ruiz Bueno, C. (2016). Evaluación de competencias profesionales en Educación Superior: Retos e implicaciones. [Evaluation of professional competences in Higher Education: Challenges and implications]. Educación XX1, 19(1), 17-38, doi:10.5944/educXX1.12175

\section{RESUMEN}

En el contexto de cambio paradigmático en la educación superior, propiciado entre otras afectaciones por el proceso de convergencia europeo en la formación de profesionales, se ha erigido el perfil y las competencias profesionales como referentes de la formación de los mismos, conllevando automáticamente nuevos planteamientos en el diseño, desarrollo y evaluación de la misma. Con independencia de los mismos, en este artículo queremos centrar la mirada sencillamente, a modo de objetivo, en cuatro retos, entre otros, que conlleva particularmente la evaluación de las competencias profesionales, que van desde diferentes dimensiones (conceptual, desarrollo-reconstructiva, estratégica y operativa) y a la vez considerar algunas implicaciones sobre los mismos.

Es por ello, la necesidad de partir previamente de una conceptualización de competencias profesionales en la actualidad, clave para afrontar no solo su formación y desarrollo, sino también su propia evaluación, dando pie a nuevas estrategias y dispositivos de evaluación de competencias profesionales sobre los que se reflexiona y especifica en el contexto de la educación superior. 


\section{PALABRAS CLAVE}

Competencia profesional; evaluación competencias; instrumentos evaluación; escenarios de evaluación; norma de competencia; educación superior.

\section{ABSTRACT}

In the context of paradigmatic change in the higher education, propitiated between other affectations for the convergence of European process in the professionals training, there have been raised the profile and the professional competences as a reference of the training of the same ones, carrying automatically news approaches in the design, development and evaluation of the same one. With independence of the same ones, in this article we want to focus, like aim, on four challenges, among others, which there carries particularly the evaluation of the professional competences, which go from different dimensions (conceptual, develop-reconstructive, strategic and operative) and simultaneously to consider some implications on the same ones.

It is for it, the need to depart before from a conceptualization of professional competences at present, fix to confront not only his training and development, but also his proper evaluation, giving course for new strategies and devices for evaluation of professional competences on those who are thought over and specify in the context of the higher education.

\section{KEY WORDS}

Professional competences; evaluation of competences; tools evaluation; context evaluation; competences rules; higher education.

\section{ACOTAMIENTOS INICIALES A MODO DE INTRODUCCIÓN}

No son pocas las publicaciones que en los últimos tiempos están emergiendo en relación con la evaluación de las competencias en general y en educación superior en particular. En la mayoría de ellas se alude a la dificultad de dicha tarea y no es de extrañar dada la cultura evaluativa de la que provenimos, básicamente ligada a lo disciplinar y a los sistemas tradicionales en los que hemos estado operando con dicho enfoque.

Para nosotros, la dificultad no deviene del propio proceso de evaluación, que también la conlleva, y la multiplicidad de finalidades, estrategias, dispositivos, instrumentos que podemos considerar. El problema se nos plantea cuando tenemos que afrontar el objeto de evaluación: las compe- 
tencias profesionales. Es en este ámbito donde encontramos verdadera dificultad conceptual que después afecta a lo estratégico-operativo. Aunque no es este el momento ni el espacio para plantearse viejos y nuevos problemas conceptuales en torno a las competencias, cabe, por imperativo personal, realizar un esfuerzo de ubicación sobre este particular, sin el cual no podremos avanzar, evitando caer en el simplismo o reduccionismo sobre la competencia desde la formación para superar el trance.

Con todo, de entrada hay que advertir que no se pueden evaluar las competencias, sino lo que denominamos competencias. Es decir, tiene que ver con la mirada que se tenga de ella. De ahí la necesidad de su conceptualización. Los conceptos no son neutros, la instrumentalización y las condiciones de evaluación tampoco (Le Boterf, 2010).

Sin lugar a duda, el cambio de paradigma provocado por el nuevo modelo de formación superior basada en competencias y con referencia a perfiles profesionales, conlleva nuevos planteamientos en el diseño, desarrollo y evaluación de dicha formación. Con independencia de los mismos, en este artículo queremos centrar la mirada sencillamente, a modo de objetivo, en cuatro retos, entre otros, que conlleva particularmente la evaluación de las competencias profesionales, que van desde diferentes dimensiones (conceptual, desarrollo-reconstructiva, estratégica y operativa) y a la vez considerar algunas implicaciones sobre los mismos.

\section{PRIMER RETO: DIMENSIÓN CONCEPTUAL. LA COMPETENCIA, UN SABER COMPLEJO: DE SABERES PARCELIZADOS A LA INTEGRACIÓN DE SABERES}

Entendemos el problema conceptual sobre la competencia y su propia evolución como «Un conjunto de conocimientos, procedimientos y actitudes combinados, coordinados e integrados en el ejercicio profesional, definibles en la acción, donde la experiencia se muestra como ineludible y el contexto es clave... Estamos apuntando en la dirección del análisis y solución de problemas en un contexto particular en el que a partir de dicho análisis (y para el mismo) se movilizan pertinentemente todos los recursos (saberes) que dispone el individuo para resolver eficazmente el problema dado» (Tejada, 1999, p. 27).

A partir de este acotamiento conceptual, nos proponemos realizar una relectura del mismo, apuntando que:

1. Una primera nota característica en el concepto de competencia es que comporta todo un conjunto de conocimientos, procedimientos y actitudes combinados, coordinados e integrados, en el sentido 
que el individuo ha de «saber», "saber hacer», «saber ser» $\mathrm{y}$ "saber estar» en relación con lo que implica el ejercicio profesional. El dominio de estos saberes le hacen «capaz de» actuar con eficacia en situaciones profesionales. Desde esta óptica, no sería diferenciable de capacidad, erigiéndose el proceso de «capacitación» en clave para el logro de las competencias. Pero una cosa es «ser capaz»y otra bien distinta es «ser competente», poseyendo distintas implicaciones idiomáticas.

De hecho, bastantes definiciones (Echeverría, 2002, 2009; Lasnier, 2000; Le Boterf, 1995, 2010; Navío, 2005; Rodríguez, Serreri, y del Cimmuto, 2010; Tardif, 2006; Tejada, 1999, 2012) así lo resaltan cuando distinguen entre el dominio o posesión de dichas características para llegar a ser capaz, lo que supone la capacidad de saber actuar. Ser competente implica disponer de un equipamiento profesional y de utilizar los recursos necesarios para desarrollar una determinada actividad. Ello implica que las competencias incluyen a las capacidades, sin las cuales es imposible llegar a ser competente, pero no se reducen a las mismas.

2. Las competencias solo son definibles en la acción. En la línea de lo apuntado anteriormente, las competencias no son reducibles ni al saber, ni al saber-hacer, por tanto no son asimilables a lo adquirido en formación. Poseer unas capacidades no significa ser competente. Es decir, la competencia no reside en los recursos (capacidades) sino en la movilización misma de los recursos. Para ser competente es necesario poner en juego el repertorio de recursos. En este sentido, saber, además, no es poseer, es utilizar, es actuar (Tardif, 2006).

Pero aún más, en esta línea argumental cabría superar una interpretación simplista de utilizar, para no quedarse en la mera aplicación de saberes. Pasar del saber a la acción es una reconstrucción: es un proceso con valor añadido. Esto nos indica que la competencia es un proceso más que un estado; es poniendo en práctica-acción la competencia como se llega a ser competente. En este sentido de actuación profesional, también podemos aludir a que la competencia está contaminada de ética (con uno mismo, con los demás, con la comunidad...). Es necesario desarrollar una base ética en la actuación de los profesionales, teniendo en cuenta su ser y el de los demás, así como el entorno ambiental. En este último punto incluso podemos hablar del componente ecológico de la competencia (saber ser y saber estar en contexto). 
3. No es suficiente con verificar qué elementos son constitutivos de las competencias. Hemos de profundizar más y de ahí que recurramos a cómo se conforman, asumiendo que no es suficiente con el proceso de formación - por ende posibilitador de las capacidades y apoyado en la formación- sino que en este terreno la experiencia se muestra como ineludible.

En síntesis, el concepto de competencia es indisociable de la noción de desarrollo. No debemos olvidar que como resultante de dicho proceso de adquisición igualmente se incrementa el campo de las capacidades entrando en un bucle continuo que va desde las capacidades a las competencias y de estas a las capacidades, iniciando de nuevo el ciclo potenciador en ambas direcciones, en un continuum inagotable («espiral centrífuga y ascendente»).

4. El contexto, por último, es clave en la definición. Si no hay más competencia que aquella que se pone en acción, la competencia no puede entenderse tampoco al margen del contexto particular donde se pone en juego. Es decir, no puede separarse de las condiciones específicas en las que se evidencia.

Estamos apuntando que en la dirección del análisis y solución de problemas en un contexto particular en el que a partir de dicho análisis (y para el mismo) se movilizan pertinentemente todos los recursos (saberes) que dispone el individuo para resolver eficazmente el problema dado.

Pero ello no quiere decir necesariamente que cada contexto exige una competencia particular, con lo cual podríamos llegar al infinito interminable de competencias, sino que la propia situación demanda una respuesta contextualizada. En este sentido, ser competente es ser capaz de actuar y reaccionar de manera pertinente y durable en una familia de situaciones (Le Boterf, 2010).

Tras esta rápida conceptualización sobre competencia profesional, podemos sintetizar en torno a:

- La competencia no es una suma/adición, sino un ensamblaje con una dinámica interaccional entre elementos.

- Hemos de tratar la competencia más como un proceso que como una suma de recursos.

- Ser competente (dominio de la acción) diferente de tener competencias (tener recursos). 
- No es suficiente con poseer los recursos —saberes- es necesario saberlos utilizar bien en contextos particulares.

Igualmente, esta conceptualización tiene sus implicaciones desde su evaluación, más concretamente como objeto de evaluación (qué evaluar). Si asumimos que la competencia profesional es un saber complejo, lo que implica todo un conjunto de saberes (conocimientos, procedimientos, actitudes), cualquier planteamiento evaluativo de la misma debe realizar un análisis cuidadoso de los mismos e integrarlos todos en dicho planteamiento. En este sentido, puede ser de utilidad considerar el modelo competencial de la Pirámide de Miller (1990) que de alguna manera, clasifica dichos saberes de cara a su evaluación en saber, saber cómo, demostrar cómo y hacer, que posteriormente retomaremos cuando abordemos la dimensión operativa de la evaluación.

En educación superior, este apunte nos remite a la consideración de la interrelación de los saberes y su integración —mapa de competencias-. Ello excede de los propósitos de este trabajo. Lo que queremos de alguna manera decir es que una competencia profesional, en muchas ocasiones, no puede adquirirse/desarrollarse en una disciplina, e incluso en un nivel o curso. Lo más probable, desde la articulación curricular, es que se aborde la misma desde distintas materias y asignaturas, incluso con graduaciones de dominio en los diferentes niveles o cursos. De hecho, desde el diseño de la formación bajo el modelo competencial, que tiene su conexión con el perfil profesional -funciones y realizaciones profesionales-, se ha optado por la consideración de unidades de competencia, igualmente en conexión con las anteriores, derivando de ellas, como referente formativo, los resultados de aprendizaje. Este abordaje también tiene sus ventajas en los procesos de evaluación de la competencia profesional por cuanto, de alguna manera, deja resuelto el qué evaluar, tomando como referencia fundamental los propios resultados de aprendizaje y los criterios de realización (De Ketele, 2006; Gerard, 2008; Laurier, 2005; Tierno, Iranzo, y Barrios, 2013).

No obstante, hay que considerar que la evaluación en educación superior, aun asumiendo el cambio de paradigma, sigue estando ligada a disciplinas, materias y asignaturas, y, en la mayoría de las ocasiones, el abordaje evaluativo se realiza desde las mismas. Si bien se tienen en cuenta los resultados de aprendizaje, desagregados de la competencia de referencia, el abordaje sigue siendo parcelizado. Es decir, podemos informar por separado de los niveles de dominio en los diferentes campos del saber, del dominio, pues, de determinados recursos, pero no de las competencias profesionales, a no ser que integremos en el proceso evaluativo al conjunto o varias disciplinas por curso. Desde esta lógica, pues, asumimos que la evaluación de la competencia profesional conlleva un planteamiento interdisciplinar con 
lo que ello supone de coordinación de materias e incluso de integración en módulos, trabajo en equipos docentes y en departamentos,...).

Caso particular es el prácticum o los trabajos de fin de grado (o máster) donde sí es posible evaluar la competencia profesional en su integridad, ya que la misma se activa en su totalidad, además de tener como referencia el conjunto de resultados de aprendizaje, no ocurriendo de esta manera en la evaluación desde las materias, que, como venimos sosteniendo, apuntan a unos determinados resultados de aprendizaje.

En síntesis de este reto, desde la conceptualización de la competencia profesional, asumimos un planteamiento complementario entre la evaluación de saberes parcelizados (recursos) que han de integrarse posteriormente y junto con los planteamientos de actuación profesional (prácticas, prácticum) y los trabajos de fin de grado o máster, poder certificar competencias profesionales. Esta es la realidad de la evaluación de la competencia profesional en educación superior: junto con información del dominio de saberes, de una parte, la integración de los mismos, añadiendo evidencias de dominio de actuación/desempeño profesional (preprofesional) a través de materias prácticas y trabajos de fin de grado, por otra.

\section{SEGUNDO RETO: DIMENSIÓN DESARROLLO-RECONSTRUCTIVA. INTEGRACIÓN ESCENARIO FORMATIVO-ESCENARIO SOCIOPROFESIONAL}

El enfoque de la formación basada en la competencia ha significado un paso adelante en el sentido de poner el énfasis en una lógica más productiva, menos académica y más orientada a la solución de problemas por encima de la reproducción de contenidos.

Este planteamiento, pues, conlleva tres dimensiones relevantes en la consideración de la formación por competencias: a) el perfil profesional como referente del diseño curricular, b) el espacio formativo, integrando la institución formativa y la institución sociolaboral y c) el tiempo formativo, que se proyecta a lo largo de toda la vida, siendo insuficiente la formación inicial y resaltando el protagonismo de la formación continua.

Partimos, como acabamos de ver, de una conceptualización de competencias que realza la acción, la experiencia y al contexto socioprofesional. Dicha conceptualización conlleva todo un conjunto de consecuencias e implicaciones en la enseñanza-aprendizaje de competencias profesionales ( $\mathrm{Te}$ jada, 2012). De momento vamos a reparar en tres de ellas asociadas a cada una de las dimensiones resaltadas. 
1. En los procesos de formación basada en competencias, los procesos de aprendizaje que se favorecen deben orientarse hacia la acción del participante tomando como referente el marco organizativo en el que la situación de socioprofesional es situación de aprendizaje. La aportación de Bunk (1994) es significativa al respecto. Para el autor, la transmisión de las competencias (mediante acciones de formación) se basa en la acción. El desarrollo de la competencia integrada (competencia de acción profesional) requiere de una formación dirigida a la acción; es decir, puede y debe relacionarse con funciones y tareas profesionales en las situaciones de trabajo con el fin de que la competencia cobre su sentido genuino y global. En la misma línea, Del Pozo (2013) considera que el desarrollo de competencias profesionales debe basarse en la acción y debe relacionarse con situaciones reales de trabajo con el fin que el desarrollo de la competencia sea significativo.

2. La consideración de los escenarios de actuación socioprofesional donde la acción y la práctica son referentes y recursos formativos. Si no hay más competencia que aquella que se pone en acción, la competencia no puede entenderse al margen del contexto particular donde se pone en juego. Es decir, no puede separarse de las condiciones específicas en las que se evidencia.

3. La experiencia es ineludible para la adquisición de las competencias y las competencias pueden ser adquiridas a lo largo de toda la vida, constituyendo, por tanto, un factor capital de flexibilidad y de adaptación a la evolución de las funciones, los requerimientos y los empleos. Esta asunción tiene que ver directamente con el propio proceso de adquisición de competencias y atribuye a las mismas un carácter dinámico.

Indiscutiblemente, el aprendizaje por competencias ha propiciado toda una renovación de las teorías psicopedagógicas de los aprendizajes, contribuyendo con ello a evolucionar los esquemas de referencia de la formación de los profesionales. Nos referimos básicamente al aprendizaje experiencial, aprendizaje situado, con sus orígenes en los propios planteamientos de Dewey, siguiendo con otros tantos como Piaget, Bruner, Kolb, Vygotsky, etc. En este sentido, cobra importancia la propia experiencia sociolaboral del que aprende, se considera que todo proceso de aprendizaje es una integración tanto de información como experiencia, reinterpretando con ello los conocimientos preexistentes, a la vez que se construyen nuevos; el aprendizaje se vuelve más efectivo al tener la referencia profesional, al estar ligado o vinculado a la resolución de dificultades o problemas reales. No solo se incrementa su percepción de utilidad y motivación en el propio proceso de 
aprendizaje, sino que también se ve incrementada su aplicabilidad. De esta forma, se fortalece la dimensión social, emocional y cognitiva de aprendizaje y desarrollo de las competencias.

La formación de competencias profesionales comporta partir de situaciones y problemas reales, propiciando diseños curriculares organizados en «core practices» (Grossmana, Hammerness \& McDonald, 2009), combinando procesos formativos externos e internos. Esto también es consecuencia de que el aprendizaje de competencias es siempre funcional; su vinculación con el contexto y la necesidad de la acción implica planteamientos metodológicos y organizativos abiertos, múltiples, variados y flexibles. Podemos afirmar que optar por un aprendizaje más reflexivo, responsable, autónomo y cooperativo facilitará la construcción significativa y el desarrollo de las competencias profesionales (Biggs, 2010; Coll, Mauri, y Rochera, 2012; Domingo y Gómez, 2014).

En este punto hemos de tener presente la referencia a situaciones auténticas de formación que son dependientes del contexto o escenario socioprofesional (Le Boterf, 2010; Lussier \& Allaire, 2004; Tejada, 2012). La adquisición de competencias toma valor, significatividad, representatividad y pertinencia a partir de las condiciones específicas en las que se activan, por lo tanto es indispensable que estas permitan la aproximación a lo real. Nos estamos refiriendo a ambientes de aprendizaje que estén centrados en:

a) situación auténtica como fuente de especificación de las competencias;

b) el estudiante como protagonista y agente de la acción;

c) el fomento de la competencia, a la acción y a los recursos;

d) la comunidad que propicia la colaboración con otros estudiantes y miembros de la misma;

e) y la evaluación auténtica, para retroalimentar en formación y para tener una valoración de su desempeño.

Hay que considerar en este punto que no siempre es posible mantener la suficiente conexión con el escenario profesional. De ahí que se realicen aproximaciones a partir de nuevas metodologías formativas basadas en la simulación (Littlewood, 2011; McGaghie, Issenberg, Petrusa \& Scalese, 2010; Ros y Conesa, 2013), al no ser posible la alternancia directa. De alguna manera, se realiza una aproximación a la realidad, aunque sea arrastrándola (conformándola) a la propia institución formativa, de manera que se pueda 
garantizar el contexto de actuación profesional, aunque sea simulado, ya que ofrece un entorno realista, seguro, rentable y flexible en el que adquirir las competencias profesionales.

Todo lo anterior nos sitúa en un escenario de cambio profundo que afecta a la organización de la docencia, las metodologías, el papel de los actores y los mismos modelos formativos, considerando la alternancia de contextos de formación como una encrucijada y complemento de la teoría con la práctica (Korthagen, 2010), cuyo análisis excede a los límites de este trabajo. No obstante, por lo que se refiere a las implicaciones en los procesos de evaluación, en el próximo reto habremos de considerarlo.

\section{TERCER RETO: DIMENSIÓN ESTRATÉGICA. NUEVAS PERSPECTIVAS DEL APRENDIZAJE, NUEVAS PERSPECTIVAS EN LA EVALUACIÓN, NUEVOS CAMINOS PARA IMPLEMENTAR LA EVALUACIÓN DE COMPETENCIAS}

Sin lugar a dudas, estamos en un cambio de paradigma realmente significativo y de gran calado en la educación superior: pasamos de la universidad del enseñar a la universidad del aprender, con todo lo que ello comporta. De la lógica disciplinar a la lógica de los perfiles profesionales y con ello en la lógica de la formación en competencias.

La formación basada en competencias en tanto metodología de exploración de saberes productivos, nos introduce de manera sistemática, en la descripción de las actividades que se aplican en la resolución de problemas vinculados a un perfil profesional determinado, en los resultados esperados y en los conocimientos que se vehiculan en ellos.

Este posicionamiento deviene de nuestra propia concepción de competencia en su doble dimensión: social y personal. En el primer caso, la dimensión social, dentro de los escenarios profesionales, aludimos a las «incumbencias» en clave de funciones que se desarrollan y le son propias a un profesional específico; en el segundo caso, la dimensión personal de la competencia, nos remite al conjunto de saberes (recursos personales) para actuar competentemente.

Queremos significar en este momento la importancia del escenario, por cuanto el mismo queda condicionado por una tecnología, una organización y una normativa, que va a circunscribir de manera significativa las funciones a acometer y los recursos a activar. Nadie discute hoy día la relevancia de estos tres constituyentes escénicos y la necesidad de su consideración a la hora de articular no solo cualquier perfil, sino lo más genuino para 
las propios propósitos de la formación de los profesionales de referencia cual es la fuente de selección de contenidos (conceptuales, procedimentales y actitudinales).

Partir de este referente en la evaluación es clave, al igual que para el diseño y desarrollo de la propia formación. En este sentido, lo que queremos decir es que es necesaria una fuerte alineación entre diseño, metodología y evaluación de la formación (Biggs, 2010; Moreno Oliver, 2014; Yáñiz y Villardón, 2006) teniendo como marco de referencia tanto el escenario como el perfil profesional, como ya hemos apuntado.

Como venimos sosteniendo, el aprendizaje en educación superior es complejo, multidimensional, por cuanto la situación de aprendizaje debe estar ligada a las actividades o realizaciones profesionales: resultados de aprendizaje, que necesita ser valorado de diferentes formas (Alsina et al., 2010; Fernández, 2010). Todo ello supera la vieja cultura de evaluación en la universidad asociada fundamentalmente a los conocimientos adquiridos, a través de dispositivos centrados en pruebas escritas y orales. Es necesario, pues, abrir la estrategia y dispositivos evaluativos lo suficiente para recoger toda la información necesaria y valorar todos los resultados esperados, en estrecha conexión con las decisiones de programación de aprendizajes realizadas y su desarrollo. Por lo tanto, la evaluación de competencias como proceso complejo debe orientarse hacia la acción del participante/profesional, tomando como referente situaciones reales y/o simuladas de trabajo que sirvan para diseñar tareas auténticas de evaluación (Del Pozo, 2013, Ashford-Rowe, Herrington \& Brown, 2014).

Además, la evaluación no solo debe medir el aprendizaje sino también favorecerlo. Desde esta lógica también entendemos la multifuncionalidad de la evaluación en el sentido de evaluación del aprendizaje, evaluación para el aprendizaje, evaluación como aprendizaje y evaluación desde el aprendizaje. Con ello recaemos y le damos sentido a la ya clásica funcionalidad formativa y sumativa de la evaluación con un enfoque complementario (Brown \& Pickford, 2013; Ion, Silva, y Cano, 2013; Nicol, Thomson \& Breslin, 2014; Villardón 2006), teniendo presente que la primera está concebida más como evaluación para el desarrollo de competencias, centrando la atención en las actividades de aprendizaje y su mejora, el feed-back continuo, la reflexión sobre el proceso, la autoevaluación y la colaboración; mientras que la segunda, la sumativa, ligada a la evaluación de las competencias, se conecta con el desempeño, realizaciones, niveles de logro, evidencias, gestión eficaz de situaciones.

Estas asunciones de cambio paradigmático evidente, conllevan automáticamente repensar el hecho evaluativo y su proceso. Es decir, desde el 
qué evaluar (objeto, indicadores, criterios,..) hasta cómo evaluar (modelos y estrategias), pasando por el para qué evaluar (finalidades y toma de decisiones), el cuándo evaluar o momento evaluativo y con qué evaluar, aludiendo a los instrumentos, técnicas o dispositivos de recogida de información y quién evaluará (agentes evaluadores).

En este sentido podemos apuntar la siguiente estrategia para la posterior concreción de los dispositivos de instrumentalización:

1. Establecimiento de las competencias o resultados de aprendizaje de los estudiantes. Esta actividad se realiza cuando se acomete el diseño del perfil profesional, a la vez que se realiza el diseño de la formación asociada. En las diferentes guías didácticas o docentes, suelen quedar reflejados, sobre todo, los resultados de aprendizaje, que son los referentes tanto para el diseño de las estrategias metodológicas, actividades de aprendizaje, como de las actividades de evaluación.

2. Identificación de situaciones de aprendizaje (profesionales) relevantes $y$ de su estructura para la evaluación de la competencia profesional. En su elaboración se llevan a cabo una secuencia de operaciones sobre los «estándares de competencia» (realizaciones profesionales, criterios de realización y contexto profesional) fijados en cada una de las unidades de competencia.

3. Extracción de los criterios de mérito. Esta operación procede de la agrupación de los criterios de realización de las unidades de competencia sobre la base de sus componentes principales, extrayendo los criterios de mérito, «esenciales» de «rendimiento», y eficacia.

4. Obtención de las evidencias suficientes de competencia. La cuarta operación consiste en la obtención de diferentes tipos de evidencias suficientes de competencia (conocimientos, destrezas y actitudes) y los métodos para su recogida, que se derivan de la resolución de las situaciones relevantes de evaluación. Se destaca aquí el trabajo de Del Pozo (2013), quien afirma que el proceso de evaluación de competencias significa obtener evidencias, que muestren comportamientos en condiciones específicas, de modo que se pueda inferir que el desempeño esperado se ha logrado de manera adecuada. Para el mismo la clave está en determinar el tipo de evidencia, su pertinencia, veracidad y vigencia.

5. Fijación de los niveles de logro de las competencias: Elaboración de escalas de competencia. La quinta operación afecta al eje de la de- 
cisión de competencia. A este respecto la elaboración de escalas descriptivas de competencia del desempeño competente referidas a cada una de las situaciones esenciales de evaluación asociadas a las unidades de competencia representa el «desempeño aceptable o umbral de competencia» a alcanzar para ser acreditado y por tanto poder sostener un argumento interpretativo en el que apoyar la emisión del juicio de competencia y poder así incrementar la consistencia en la interpretación de los estándares de competencia.

Un tema particular en este contexto de análisis es el de los criterios de evaluación.

La lógica de la competencia nos lleva a la lógica de la norma o los estándares competenciales (Jornet, González, Suárez, y Perales, 2011). De hecho, en la mayoría de las experiencias de evaluación de esta naturaleza, se fijan dichos estándares con distintos formatos. Por ejemplo, podemos tener un referente criterial asociado directamente a niveles de desempeño: satisfactorio, básico, competente o destacado, o no satisfactorio, satisfactorio o sobresaliente. En ambos casos se alude a la presencia de los criterios de realización.

En otros casos, puede aludirse a niveles de normalización (básico, medio o avanzado) o también novel, junior, sénior, experto. En estos casos, dichos criterios apuntan más directamente en la dirección del desarrollo profesional en clave de carrera profesional, ya que se explicita más directamente, por su propia nomenclatura, el mismo y sus implicaciones sociolaborales, organizativas, formativas, ...

Asumimos como evidencias las pruebas claras y manifiestas de los conocimientos, habilidades, destrezas y actitudes que una persona posee y que determinan su competencia. Las mismas pueden clasificarse de diferentes formas. En el contexto de este trabajo vamos a agruparlas en dos:

- Evidencia de conocimiento: incluye el conocimiento de lo que tiene que hacerse, el cómo habría de hacerlo, el por qué tendría que hacerse y lo que habría que hacer si las condiciones del contexto cambiaran en el desarrollo de la actividad. Implica la posesión de un conjunto de conocimientos, teorías, principios y habilidades cognitivas que permiten contar con un punto de partida y un sustento para un desempeño eficaz.

- Evidencia de desempeño: refiere el comportamiento por sí mismo y consiste en descripciones sobre variables o condiciones que permiten inferir que el desempeño fue efectivamente logrado. Pueden ser 
obtenidas de forma directa o por el producto. En el primer caso, permite apreciar de manera más objetiva y directa el resultado de aprendizaje; en el segundo, es un resultado tangible de la actividad realizada y su valoración se fundamenta en las condiciones del criterio de desempeño.

Pero el problema fundamental de las evidencias no es su tipología, sino fundamentalmente su determinación en clave de qué conocimientos, destrezas o habilidades se desean medir, cuántas evidencias se requieren, cuántos tipos diferentes de evidencias,... En este sentido, lo importante es generar las condiciones para hacer visible y disponible todo aquello que se aprende.

Esto obliga a determinar qué tipos de actividades permitirán reunir evidencia, lo cual nos remite a la cantidad y calidad suficiente de las evidencias que debemos recoger. Privilegiar la selectividad de «situaciones de aprendizaje auténticas» (un riesgo a tomar) frente a la exhaustividad en la confección de referentes de competencias, tentación explicable, pero no se pueden acotar todas las actividades o todas las situaciones profesionales.

La evidencia suficiente se define como el mínimo imprescindible que debe ser evaluado. Las evidencias suficientes de desempeño son las generadas por las personas en la resolución de las situaciones profesionales.

Sea como fuere hemos de tener presentes algunos criterios y principios para valorar las evidencias previas o actuales durante el proceso de evaluación de las competencias profesionales, que deben aplicarse integralmente y no son excluyentes. Respecto de los primeros, nos referimos a pertinencia, en el sentido que las evidencias deben corresponder con las requeridas en la norma de competencia; vigencia o actualidad, en el sentido de garantizar que no son obsoletas, y autenticidad, para poder garantizar que corresponden a una persona evaluada y no otra, que las aportó solo y no con la participación de otras personas. Respecto de los segundos, hay que usar métodos de evaluación más adecuados para evaluar la competencia de manera integrada, seleccionar los métodos que sean más directos y relevantes para aquello que se está evaluando, y por último, usar una amplia base de evidencias para inferir la competencia.

La aplicación de la evidencia «necesaria» para la evaluación de la competencia implica la necesidad de instrumentar un sistema de evaluación, mediante la identificación de criterios e indicadores que permitan evaluar las evidencias recogidas en situaciones esenciales y auténticas de evaluación (Lussier y Allaire, 2004). 


\section{CUARTO RETO: DIMENSIÓN OPERATIVA: VALIDEZ/PERTINENCIA DE LOS DISPOSITIVOS: ESCALANDO LA PIRÁMIDE DE MILLER}

La evaluación de competencias en educación superior exige también de la articulación de dispositivos válidos y fiables donde se pueda evidenciar que la misma se posee, aunque no debemos olvidar que la competencia no puede ser observada directamente, sino inferida por el desempeño o acciones específicas.

Partimos de la consideración de que no hay ningún método o instrumento de evaluación por sí solo que pueda proporcionar toda la información para juzgar la competencia de un profesional. Partimos del principio de la multivariedad y triangulación instrumental, que nos lleva a integrar y, por tanto, conjugar coherentemente diferentes modos de recoger evidencias de la competencia profesional (Cano, 2008; De Ketele, 2006; Gerard, 2008).

Por otra parte, como venimos sosteniendo, la competencia solo se puede evaluar en la acción, si bien para su adquisición y desarrollo cabe haber adquirido previamente toda una serie de saberes (conocimientos, habilidades y actitudes), que hemos de describir de acuerdo a los resultados de aprendizaje o norma de competencia. En consonancia con la pirámide de Miller (1990), podemos acometer la evaluación de la competencia a partir de los distintos tipos de saberes (Tejada, 2011). Siguiendo la misma, podemos aludir a cuatro niveles de evaluación en relación directa del tipo de saber, correspondiendo los dos primeros propiamente al conocimiento y los siguientes, desde la misma lógica, al comportamiento.

En la base de la pirámide estaría el saber (know) o conjunto de conocimientos teóricos que todo profesional debe dominar como fundamentadores de la práctica profesional, a sabiendas, como indica el propio Miller, que no son suficientes, y por tanto no podemos quedarnos en este nivel de evaluación para atrapar la competencia, sencillamente estaríamos en su dimensión más cognitiva.

En el segundo nivel, estaría el saber cómo (know how) usaría los saberes teóricos adquiridos si los tuviera que poner en práctica en un contexto particular. En este nivel evaluativo podemos obtener una pre-visión, en el sentido de visión previa de la práctica profesional.

En el tercer nivel, nos encontraríamos con el demuestra cómo (show how) lo haría. Es una demostración con hechos, cercana a la realidad, pero aún no es la práctica profesional directa. Las simulaciones (role playing) pueden ser buenas situaciones a tal fin, pero con ello tampoco podemos concluir sobre la competencia profesional. 
En el cuarto nivel, en la cúspide de la pirámide, Miller incardina el hacer (doing) en la práctica profesional. Estaríamos pues ante una actuación real, en un contexto o situación profesional de desempeño y en el momento de evidenciar la competencia.

En la cúspide, nosotros nos hemos atrevido a añadir un quinto nivel que tiene que ver con hace y enseña, (doing and teaching), es decir, desempeña con maestría. En dicho nivel integramos, pues, de alguna manera, nuestros niveles de dominio experto apuntados con anterioridad como norma de competencia, que además son útiles en la dirección de desarrollo profesional en clave de carrera profesional y su evaluación.

Aunque pudieran parecer excesivas las exigencias del modelo, son necesarios e imprescindibles todos los niveles, por cuanto no debemos quedarnos solo en la activación de la competencia en el escenario profesional, sino que debemos tener también presente con qué tipo de equipamiento (recursos) se accede a dicho escenario profesional, como ya hemos anticipado.

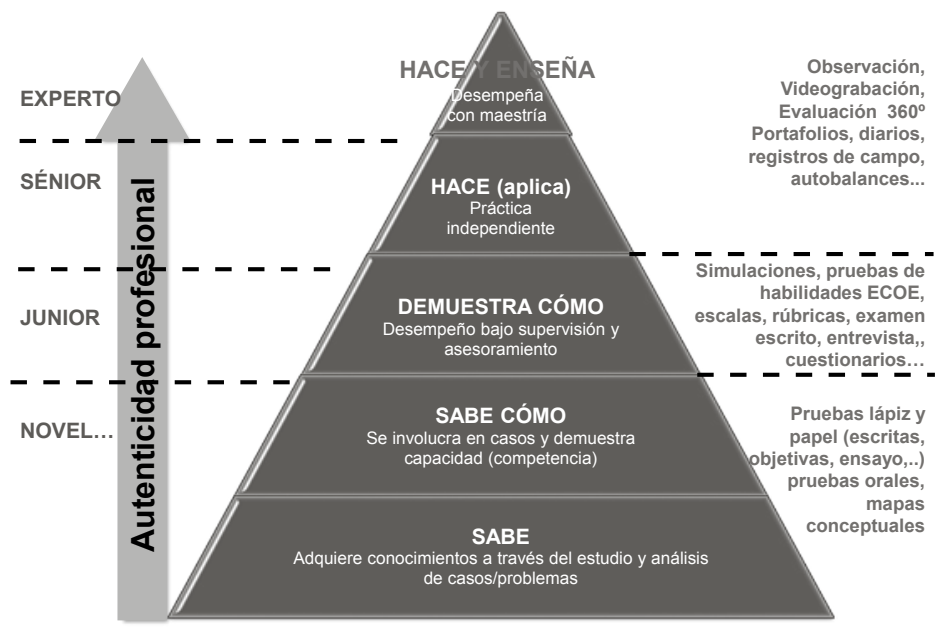

Figura 1. Tipología de dispositivos según tipología de saberes (elaboración propia a partir de la Pirámide de Miller, 1990)

Esta descripción, aunque útil para nuestros propósitos de evaluación de la competencia, no la consideramos definitiva, por cuanto no integra explícitamente los saberes asociados al saber ser y saber estar (actitudes, valores y normas fundamentalmente), aunque pueden considerarse implícitos en el saber actuar en contexto, en la línea de Tejada (2012). 
Su interés está en la consideración de dos grandes bloques de pruebas o instrumentos de evaluación en consonancia con la propia estructura de la pirámide cognición-comportamiento: qué conocimientos sabe y sabe cómo usar los conocimientos a través de pruebas teóricas y prácticas, exámenes, entrevistas,..; muestra cómo lo haría a través de portafolios, carpetas de aprendizaje, proyectos, análisis de simulaciones, role-playing,...; qué hace y enseña a través de escalas de observación, rúbricas, estructuración de la práctica profesional ... En otras palabras, podríamos aludir a una evaluación tradicional, referida a las típicas pruebas de lápiz y papel en conexión con el ámbito cognitivo, y a una evaluación de ejecuciones, más variada y más posibilista para atrapar un rango mayor de competencias a través de productos, resolución de problemas reales, prácticas profesionales, autoevaluación, diarios, evaluación por colegas, evaluación $360^{\circ}$, balance y autobalance de competencias...

Dados los límites de este trabajo, obviamos profundizar en una descripción detallada de la multivariedad de instrumentos para llevar a cabo la evaluación de la competencia de acción profesional en los escenarios profesionales. En este sentido, nos remitimos a un trabajo anterior, sobre los dispositivos e instrumentos de evaluación de competencias.

Sirva como reflexión final de este apartado que la selección y uso de instrumentos de evaluación está relacionado con qué y cuánta evidencia es suficiente para evaluar la competencia. A ello, también, hay que añadir el nivel de precisión y la cantidad de riesgo que es aceptable. Es decir, si queremos ser precisos y correr pocos riesgos, el dispositivo instrumental debe ser amplio y multivariado, a la par que hay que garantizarle validez, confiabilidad, flexibilidad e imparcialidad.

\section{CONSIDERACIONES FINALES A MODO DE CONCLUSIÓN}

Dadas las características de la competencia profesional y su complejidad, no cabe duda que al igual que no existe un único instrumento, como hemos apuntado, capaz de atraparla, tampoco existe un agente evaluador único que así lo pueda hacer. Estamos diciendo sencillamente que la competencia profesional implica procesos de autoevaluación, heteroevaluación y coevaluación en consonancia directa con los sistemas de registro que acabamos de señalar.

Con esta asunción de procesos de auto-hetero-coevaluación no resolvemos el problema de los agentes evaluadores, sino sencillamente que destacamos a través de qué procesos hacer. Los agentes evaluadores a los que nos referimos hay que buscarles en el propio escenario de actuación profesional 
donde se activa, expresa y evidencia la competencia profesional. En dicho escenario encontramos profesionales y otros implicados con diferentes funciones, roles y responsabilidad profesional: nos referimos a profesores, directivos, tutores, subordinados, iguales o pares, alumnos, otros miembros de la institución,...

Por tanto, habrá que integrar a través de procesos de triangulación a tales agentes evaluadores. Hay que advertir, en cualquier caso, que no todos los protagonistas tienen el mismo peso e incidencia en el proceso de evaluación. Habrá pues, que determinar o ponderar tanto el papel, el valor y significado de la información aportada, etc. a la hora de integrar la misma y tomar decisiones sobre el particular. En síntesis, este nuevo enfoque reclama procesos más participativos y democráticos e incluye la utilización de mecanismos para llegar a acuerdos e integrar las diferentes perspectivas valoradas.

Por último, y a modo de síntesis, queremos concluir que la evaluación de competencias en educación superior, además de lo comentado, retoma el sentido formativo y se constituye en una oportunidad de aprendizaje y desarrollo, a la par que tiene una función autorreguladora, al hacer más conscientes a los estudiantes de su nivel. De ahí que haya que abordarla con un enfoque más videográfico que fotográfico. Coll, Mauri \& Rochera (2012), en este sentido, concluyen que la evaluación de competencias, su diseño e implementación, ha de fomentar la implicación y responsabilidad del alumno y la mejora de su propio proceso de aprendizaje.

Con todo, no hay que olvidar que la evaluación de la competencia profesional conlleva aspectos positivos y negativos (Duta et al. 2009; Tierno, Iranzo \& Barrios (2013), destacando entre los primeros la mejora del aprendizaje, la coherencia profesional, satisfacción e implicación, la integración, la formación docente e innovación, así como la mejora de la comunicación entre los docentes. Por el contrario, también hay que apuntar nuevas cargas de trabajo, más tiempo en el proceso de evaluación, resistencias al cambio, dificultades de coordinación, tamaño de los grupos y condiciones logísticas y la ausencia de formación del profesorado (Ión \& Cano, 2012; Margalef, 2014).

En relación con estos segundos, no cabe duda que hay que cambiar la cultura evaluativa institucional, además de integrar las potencialidades de las TIC que pueden no solo aliviar la tarea evaluativa, sino convertirse en herramientas clave para el desarrollo y gestión de los procesos evaluativos de competencias profesionales. 


\section{REFERENCIAS BIBLIOGRÁFICAS}

Alsina, J. (Coord.); Boix, R.; Burset, S.; Buscà, F.; Colomina, R. M; Garcia, A.; Mauri, T.; Pujolà, J. T. y Sayós, R. (2010). Avaluació per competències a la universitat: les competències transversals. Barcelona: ICE Universidad Barcelona-Octaedro.

Arafeh. J. M. R. (2011). Simulation-based Training. Journal of Perinatal and Neonatal Nursing, 25 (2), 171-174, https:// www.nursingcenter.com/_PDF_.asp $\mathrm{x}$ ? $\mathrm{an}=00005237-201104000-00018$. DOI:10.1097/JPN.0b013e3182116e55

Ashford-Rowe, K.; Herrington, J. \& Brown, Ch. (2014). Establishing the critical elements that determine authentic assessment. Assessment \& Evaluation in Higher Education. 39(2) 205-222. DO I:10.1080/02602938.2013.819566

Biggs, J. (2010). Calidad del aprendizaje universitario. (4 ed). Madrid: Narcea.

Bunk, G.P. (1994). La transmisión de competencias en la formación y perfeccionamiento profesionales de la RFA. Revista Europea de Formación Profesional. 1, 8-14.

Cano, E. (2008). La evaluación por competencias en la educación superior. Profesorado. Revista de currículum y formación del profesorado, 12,3. http:// www.ugr.es/local/recfpro/rev123col1. pdf

Cano, E. (Coord.) (2011). Buenas prácticas en la evaluación de competencias. Cinco casos de educación superior. Barcelona: Laertes.

Coll, C; Mauri, T., y Rochera, M.J. (2012). La práctica de evaluación como contexto para aprender a ser aprendiz competente. Profesorado. Revista de curriculum y formación del profesorado, 16 (1), 50-57.
De Ketele, J. M. (2006). Caminhos para a avaliaçao de competencias, Revista Portuguesa de Pedagogia, 40,3, 135-147.

Del Pozo, J. A. (2013). Competencias profesionales. Herramientas de evaluación: el portafolios, la rúbrica y las pruebas situacionales. Madrid: Narcea.

Domingo, A. y Gómez, V. (2014). La práctica reflexiva. Bases, modelos e instrumentos. Madrid: Narcea.

Duta, N; Compaño, P; Cárdenas, J. y Urriola, K. (2009). Aspectos positivos y negativos en la experiencia de evaluación por competencias de los docentes universitarios. En M. T. Tortosa, JD. Álvarez y N. Pellin (Coords.) VII Jornadas de Redes de Investigación en docencia universitaria. (pp. 422-430). Alicante: ICE de la Universidad de Alicante.

Echeverría, B. (2002). Gestión de la competencia de acción profesional. Revista de Investigación educativa, 20 (1), 7-43.

Echeverría, B. (2009). Trece interrogantes sobre la E. R. A. de las competencias, en J. Tejada, (coord.) et al. Estrategias de innovación en la formación para el trabajo. (pp. 331-346). Madrid: Tornapunta.

Fernández, A. (2010). La evaluación orientada al aprendizaje en un modelo de formación por competencias en la educación universitaria. REDU. Revista de Docencia Universitaria. 8 (1) 11-34.

Gerard, F. M. (2008). Evaluer competences. Guide pratique, Bruxelles: De Boeck

Grossmana, P.; Hammerness, K. \& McDonald, M. (2009). Redefining teaching re-imagining teacher education. Teachers and Teaching: 
theory and practice, 15(2), 273-289. DOI:10.1080/13540600902875340

Ion, G.; Silva, P. y Cano, E. (2013). El feedback y el feedforward en la evaluación de las competencias de estudiantes universitarios. Profesorado. Revista de currículum, y formación del profesorado.17 (2)

Ion, G. y Cano, E. (2012). La formación del profesorado para la implementación de la evaluación por competencias. Educación XX1, 15(2) 249-270. DOI: 10.5944/educxx1.15.2.141

Jornet, P.; González, J.; Suárez, J. M. y Perales, M. J. (2011). Diseño de procesos de evaluación de competencias: consideraciones acerca de los estándares en el dominio de las competencias. Bordón, 63(1) 125-145

Korthagen, F. A. (2010). La práctica, la teoría y la persona en formación del profesorado. Revista Interuniversitaria de Formación del Profesorado. 68 $(24,2), 88-101$.

Laurier, M. D. (2005). Évaluer les competences: pas si simple. Formation et Profession 11(I) 14-17.

Lasnier, F. (2000). Réussir la formation par competénces. Montreal: Guerin

Le Boterf, G. (1995). De la competénce: essai sur un attracteur étrange. Paris: Les Editions d'Organisations.

Le Boterf, G. (2010). Repenser la compétence. Paris: Editions d'Organisation.

Littlewood, K. (2011). High fidelity simulation as a research tool. Best practice \& Research Clinical Anaesthesiology, 25, 473-487. DOI:10.1016/j. bpa.2011.08.001

Lussier, O. \& Allaire, H. (2004). Lévaluation "authentique». Pedagogie collégiale, 17 (3), 29-30.
Margalef García, L. (2014). Evaluación formativa de los aprendizajes en el contexto universitario: Resistencias y paradojas del profesorado. Educación XX1, 17 (2), 35-55. DOI: 10.5944/edu$\operatorname{cxx} 1.17 .2 .11478$

McGaghie, W. C, Issenberg, S. B., Petrusa, E. R., \& Scalese, R. J. (2010). A critical review of simulation-besed medical education research: 20032009. Medical education. 44, 50-63. DOI:10.1111/j.1365-2923.2009.03547.x

Miller, G. E. (1990). The assessment of clinical skills/competence/performance/ Academic Medicine, 65 (9), s63-s67. DOI:10.1097/00001888199009000-00045

Moreno Oliver, V. (2014). Análisis y propuesta de intervención sobre la alineación entre el trabajo por competencias, la estrategia metodológica y el sistema de evaluación (Alineación $C^{*} M * E$ ). Tesis Doctoral. Dpto. Pedagogía Aplicada. Universitat Autònoma de Barcelona.

Navío, A. (2005). Las competencias profesionales del formador. Barcelona: Octaedro.

Nicol, D.; Thomson, A. \& Breslin, C. (2014). Rethinking feedback practices in higher education: a peer review perspective. Assessment \& Evaluation in Higher Education. 39 (1) 102-122. DOI:10.1080/02602938.2013.795518

Rodríguez, M. L., Serreri, P. y Del Cimmuto, A. (2010). Desarrollo de competencias: Teoría y práctica. Barcelona: Laertes educación.

Ros, M. y Conesa, C. (2013). Adquisición de competencias a través de la simulación y juego de rol en el área contable. Estudios sobre el Mensaje Periodístico, 19, número especial, 419-428. DOI:10.5209/rev_ESMP.2013. v19.42049 
Tardif, J. (2006). L'evaluation des compétences. Documenter le parcours de developpment. Montreal: Cheneriele Education.

Tejada, J. (1999). Acerca de las competencias profesionales I, Herramientas, $56,20-30$.

Tejada, J. (2011). La evaluación de competencias en contextos no formales: Dispositivos e instrumentos de evaluación. Revista de Educación. 354, 731-745. DOI:10.4438/1988-592XRE-2011-354-018

Tejada, J. (2012). La alternancia de contextos para la adquisición de competencias profesionales en escenarios complemen- tarios de educación superior: marco y estrategia. Educación XX1, 15 (2), 17-40. DOI:10.5944/educxx1.15.2.125

Tierno; J. Iranzo, P. y Barrios, Ch. (2013). El compromiso organizativo e institucional para diseñar y evaluar competencias en la universidad. Revista de Educación, 361, 223-251. DOI: 10.4438/1988-592X-RE-2011-361-141

Villardón, L. (2006) Evaluación del aprendizaje para promover el desarrollo de competencias. Educatio Siglo $X X I, 24,57-76$.

Yáñiz, C. y Villardón, L. (2006). Planificar desde las competencias para promover el aprendizaje. Bilbao: Mensajero. 